\title{
On attributes of a Rotating Neutron star with a Hyperon core
}

\author{
T. K. Jha*, H. Mishra ${ }^{\dagger}$ and V. Sreekanth ${ }^{\ddagger}$ \\ Theoretical Physics Division, Physical Research Laboratory, Navrangpura, Ahmedabad, India - 380009
}

(Dated: August 29, 2018)

\begin{abstract}
We study the effect of rotation on global properties of neutron star with a hyperon core in an effective chiral model with varying nucleon effective mass within a mean field approach. The resulting gross properties of the rotating compact star sequences are then compared and analyzed with other theoretical predictions and observations from neutron stars. The maximum mass of the compact star predicted by the model lies in the range $(1.4-2.4) M_{\odot}$ at Kepler frequency $\Omega_{K}$, which is consistent with recent observation of high mass stars thereby reflecting the sensitivity of the underlying nucleon effective mass in the dense matter EoS. We also discuss the implications of the experimental constraints from the flow data from heavy-ion collisions on the global properties of the rotating neutron stars.
\end{abstract}

PACS numbers: 21.65.+f, 13.75.Cs, 97.60.Jd, 21.30.Fe, 25.75.-q, 26.60.+c

\section{INTRODUCTION}

Recent advances in the study of cold dense matter has received new impetus both from observational astronomy and the recent heavy-ion collision experiment as well as the upcoming compressed baryonic matter (CBM) experiments, that can put constraints on the equation of state (EoS) of dense matter [1]. Arguably the uncertainties in the dense matter EoS can also be reduced appreciably through theoretical modeling of neutron stars and by analyzing their global properties with an eye on the observational data from these compact objects. Consequently, building an EoS in accordance with the heavy-ion collision data and its subsequent application to the dense matter problem seems to be prerequisite. The study can throw significant light on potential problems such as the isospin dependence of nuclear forces, the presence of stable hyperon in neutron star core and its impact on cold dense matter [2, 3].

Lately, there has been some striking signatures or observations of both massive and the low mass stars, which may have interesting implications on the structure and composition of neutron stars [4]. For example, the observation of massive compact stars $\left(M \approx 2 M_{\odot}\right)[\underline{5}, 6]$ from observation of QPO's (Quasi Periodic Oscillations) from X-ray emmisions on one hand and also the least massive ones $1.18 \pm 0.02 M_{\odot}$ [7] from the binary pulsar $J 1756-2251$ emphasize the need to address key issues in limiting the observed global properties of the stars in th $M-R$ plane. On the contrary, the canonical value of $M=1.44 M_{\odot}$, the largest precisely measured mass of the Neutron star PSR1913 + 16 [8] effectively doesn't seem to provide any stringent condition and most of the existing realistic equation of state satisfies the criteria. These aforesaid facts signifies the need to constraint the

\footnotetext{
*email: tkjha@prl.res.in

†email: hm@prl.res.in

‡email: skv@prl.res.in
}

nuclear EoS of dense matter which are extrapolations of the properties defined in the vicinity of nuclear saturation density and therefore it is important to test these extrapolations with regard to the available experimental flow data from heavy-ion collisions.

Equivalently considerable emphasis is being laid to understand nuclear matter EoS in the density range of $(1-3) \rho_{0}$, the region from where the entire possibilities of exotic forms of matter (hyperons, condensates of bosons or quarks) 9, 10, 11, 12, 13] starts appearing. It is known that the neutron star radius is sensitive to the properties of nuclear force in the immediate vicinity of nuclear saturation density $\left(\rho_{0}\right)$, whereas the maximum mass of the star is regulated by the dense matter EoS well beyond $2 \rho_{0}$ [13]. Among the various scenarios related to the star structure, the presence of stable hyperons is an exciting possibility and is known to have significant impact on the dense matter equation of state and in turn gets manifested in the star properties too [10, 14, 15]. The hyperons such as $\Lambda^{0}(1116), \Sigma^{-, 0,+}(1193)$ and $\Xi^{-, 0}(1318)$ appear at the expense of nucleons and forms a sizeable population in the density region of current interest. Although hypernuclei experiments [16] supports the presence of hyperons in dense matter, however this domain is also marred by uncertainties revolving around the nature of hyperon-hyperon interactions and their coupling strengths. Since they are the dominant species in the dense matter population, there is even greater need to understand the nature and behavior of these interactions.

Apart from that, it is also known that the addition of quartic terms softens the EoS considerably in addition to that caused by the hyperons, which thereby makes it difficult to obtain a neutron star mass larger than $2 M_{\odot}$ [17]. Here in the present investigation, we employ a model which has chiral symmetry and also embodies a dynamical generation of the vector meson mass along with the non-linear term in the scalar field interaction. The model has been used extensively to dense matter studies [18, 19, 20], which was then modified with the addition of non-linear terms in the scalar field interaction to reproduce nuclear saturation properties at rea- 
sonable incompressibility 21, 22]. Finally we generalized the model to include the lowest lying octet of baryons in our previous work [23] and applied it to study neutron star matter with varying incompressibility and varying nucleon effective mass. From our previous analysis [23], it was apparent that the difference in the nuclear incompressibility was neither prominent in the EoS, nor in the static star properties, whereas the EoS was extremely sensitive to the underlying nucleon effective masses and hence was also visible in the global properties of the star. In continuation of our earlier work, we now extend our analysis to study the rotational attributes of neutron star with varying nucleon effective mass in the mean-field approach within the same hadronic model. In short, our motivation here has been to investigate how the rotational attributes of the neutron star restrict the parameters of the present model.

The outline of the paper is as follows: In the next section, we give a brief description of the ingredients of the hadronic model that we implement in our present work. After describing the numerical scheme for calculating the rotating neutron star sequences, the general features of the equation of state of hyperon rich matter is studied and the global properties of the rotating neutron star is presented. We shall analyze these results with respect to varying nucleon effective mass and also talk of a few constraints on the neutron star mass and radius imposed by recent observations. Finally we conclude with outlook on the possible extensions of the current approach.

\section{THE EQUATION OF STATE}

In this context, we tried to explore the consequences of an EoS based on chiral $\sigma$ model with nonlinear interaction terms in the scalar field. To have a realistic description of the dense neutron star matter, we consider the effective Lagrangian of the chiral model generalized to include the lowest lying octet of baryons $\left(n, p, \Lambda^{0}, \Sigma^{-, 0,+}, \Xi^{-, 0}\right)$ interacting through the exchange of the pseudo-scalar meson $\pi$, the scalar meson $\sigma$, the vector meson $\omega$ and the iso-vector $\rho$-meson, and is given by [23]:

$$
\begin{aligned}
\mathcal{L}= & \bar{\psi}_{B}\left[\left(i \gamma_{\mu} \partial^{\mu}-g_{\omega B} \gamma_{\mu} \omega^{\mu}-\frac{1}{2} g_{\rho B} \vec{\rho}_{\mu} \cdot \vec{\tau} \gamma^{\mu}\right)-g_{\sigma B}\left(\sigma+i \gamma_{5} \vec{\tau} \cdot \vec{\pi}\right)\right] \psi_{B} \\
& +\frac{1}{2}\left(\partial_{\mu} \vec{\pi} \cdot \partial^{\mu} \vec{\pi}+\partial_{\mu} \sigma \partial^{\mu} \sigma\right)-\frac{\lambda}{4}\left(x^{2}-x_{0}^{2}\right)^{2}-\frac{\lambda B}{6}\left(x^{2}-x_{0}^{2}\right)^{3}-\frac{\lambda C}{8}\left(x^{2}-x_{0}^{2}\right)^{4} \\
& -\frac{1}{4} F_{\mu \nu} F_{\mu \nu}+\frac{1}{2} g_{\omega B}{ }^{2} x^{2} \omega_{\mu} \omega^{\mu}-\frac{1}{4} \vec{R}_{\mu \nu} \cdot \vec{R}^{\mu \nu}+\frac{1}{2} m_{\rho}^{2} \vec{\rho}_{\mu} \cdot \vec{\rho}^{\mu}
\end{aligned}
$$

The first line of the above Lagrangian represents the interaction of baryons $\Psi_{B}$ with the aforesaid mesons. In the second line we have the kinetic and the non-linear terms in the pseudoscalar-isovector pion field ' $\vec{\pi}$ ', the scalar field ' $\sigma$ ', and with $x^{2}=\vec{\pi}^{2}+\sigma^{2}$. Finally in the last line, we have the field strength and the mass term for the vector field ' $\omega$ ' and the iso-vector field ' $\vec{\rho}$ meson. The terms in eqn. (1) with the subscript ' $B$ ' should be interpreted as sum over the states of all baryonic octets. In this paper we shall be concerned only with the normal non-pion condensed state of matter, so we take $\langle\vec{\pi}\rangle=0$.

The interaction of the scalar and the pseudoscalar mesons with the vector boson generates a dynamical mass for the vector bosons through spontaneous breaking of the chiral symmetry with scalar field getting the vacuum expectation value $x_{0}$. Then the masses of the baryons, the scalar and the vector mesons, are respectively given by

$$
m_{B}=g_{\sigma B} x_{0}, \quad m_{\sigma}=\sqrt{2 \lambda} x_{0}, \quad m_{\omega}=g_{\omega B} x_{0} .
$$

In the above, $x_{0}$ is the vacuum expectation value of the $\sigma$ field. We could have taken an interaction of the $\rho$-meson with the scalar and the pseudoscalar mesons similar to the omega meson. However, a dynamical mass generation mechanism of the $\rho$-meson in a similar manner will not generate the correct symmetry energy. Therefore, we have taken an explicit mass term for the isovector $\rho$-meson similar to what was considered in earlier works 20, 21, 22, 23].

We employ the mean-field procedure to evaluate the meson fields in our present calculations. In the meanfield treatment, one assumes the mesonic fields to be uniform i.e., without any quantum fluctuations. We recall here that this approach has been extensively used to obtain field-theoretical EoS for high density matter [19], and gets increasingly valid when the source terms are large [24]. The details of the model that we use in our present investigation and its attributes such as the derivation of the equation of motion of the meson fields and its equation of state $(\varepsilon \& P)$ of the many baryonic system, can be found in our preceding work [23]. For the sake of completeness however we write down the meson field equations. The vector and the iso-vector fields are 
respectively given by

$$
\begin{gathered}
\omega_{0}=\sum_{B} \frac{\rho_{B}}{g_{\omega B} x^{2}}, \\
\rho_{03}=\sum_{B} \frac{g_{\rho B}}{m_{\rho}^{2}} I_{3 B} \rho_{B} .
\end{gathered}
$$

In the above equations the quantity $\rho_{B}$ is the Baryon density and $I_{3 B}$ is the $3 \mathrm{rd}$-component of the isospin of each baryon species.

The scalar field equation can be written in terms of the variable $Y=x / x_{0}$ with $\left.x=\left(<\sigma^{2}+\pi^{2}\right\rangle\right)^{1 / 2}$ as [23]

$$
\sum_{B}\left[\left(1-Y^{2}\right)-\frac{B}{c_{\omega B}}\left(1-Y^{2}\right)^{2}+\frac{C}{c_{\omega B}^{2}}\left(1-Y^{2}\right)^{3}+\frac{2 c_{\sigma B} c_{\omega B} \rho_{B}^{2}}{m_{B}^{2} Y^{4}}-\frac{2 c_{\sigma B} \rho_{S B}}{m_{B} Y}\right]=0
$$

where the effective mass of the baryonic species is $m_{B}^{\star} \equiv$ $Y m_{B}$ and $c_{\sigma B} \equiv g_{\sigma B}^{2} / m_{\sigma}^{2}$ are the $c_{\omega B} \equiv g_{\omega B}^{2} / m_{\omega}^{2}$ are the usual scalar and vector coupling constants respectively. It may be noted that the parameter ' $\lambda$ ' in the Lagrangian does not appear explicitly in eqn. (5), but enters implicitly through the mass term for the scalar meson, following equation (2). Similarly, in the present model describing dense matter, the $\omega$-meson mass is generated dynamically. This vector meson mass enters in Eq. (5) through the ratio $c_{\omega}=\left(g_{\omega} / m_{\omega}\right)^{2} \equiv 1 / x_{0}{ }^{2}$ which has to be fixed alongwith $c_{\sigma}$ so as to satisfy the nuclear matter saturation properties, similar to what has been done in earlier works [20, 21, 22, 23]. Further in Eq.(5), the quantities $\rho_{B}$ and $\rho_{S B}$ are the baryon density and the scalar density for a given baryon species given respectively as,

$$
\begin{gathered}
\rho_{B}=\frac{\gamma}{(2 \pi)^{3}} \int_{o}^{k_{B}} d^{3} k \\
\rho_{S B}=\frac{\gamma}{(2 \pi)^{3}} \int_{o}^{k_{B}} \frac{m_{B}^{*} d^{3} k}{\sqrt{k^{2}+m_{B}^{\star 2}}}
\end{gathered}
$$

where $k_{B}$ is the fermi momentum of the baryon and $\gamma=2$ is the spin degeneracy factor.

We now go directly to the total energy density ' $\varepsilon$ ' and pressure ' $P$ ' for a given baryon density in terms of the dimensionless variable $Y=x / x_{0}$ which is given as:

$$
\begin{aligned}
\varepsilon & =\frac{2}{\pi^{2}} \int_{0}^{k_{B}} k^{2} d k \sqrt{k^{2}+m_{B}^{\star 2}}+\frac{m_{B}^{2}\left(1-Y^{2}\right)^{2}}{8 c_{\sigma B}}-\frac{m_{B}^{2} B}{12 c_{\omega B} c_{\sigma B}}\left(1-Y^{2}\right)^{3} \\
& +\frac{m_{B}^{2} C}{16 c_{\omega B}^{2} c_{\sigma B}}\left(1-Y^{2}\right)^{4}+\frac{1}{2 Y^{2}} c_{\omega_{B}} \rho_{B}^{2}+\frac{1}{2} m_{\rho}^{2} \rho_{03}^{2}+\frac{1}{\pi^{2}} \sum_{\lambda=e, \mu^{-}} \int_{0}^{k_{\lambda}} k^{2} d k \sqrt{k^{2}+m_{\lambda}^{2}}, \\
P & =\frac{2}{3 \pi^{2}} \int_{0}^{k_{B}} \frac{k^{4} d k}{\sqrt{k^{2}+m_{B}^{\star 2}}}-\frac{m_{B}^{2}\left(1-Y^{2}\right)^{2}}{8 c_{\sigma B}}+\frac{m_{B}^{2} B}{12 c_{\omega B} c_{\sigma B}}\left(1-Y^{2}\right)^{3} \\
& -\frac{m_{B}^{2} C}{16 c_{\omega B}^{2} c_{\sigma B}}\left(1-Y^{2}\right)^{4}+\frac{1}{2 Y^{2}} c_{\omega_{B}} \rho_{B}^{2}+\frac{1}{2} m_{\rho}^{2} \rho_{03}^{2}+\frac{1}{3 \pi^{2}} \sum_{\lambda=e, \mu^{-}} \int_{0}^{k_{\lambda}} \frac{k^{4} d k}{\sqrt{k^{2}+m_{\lambda}^{2}}}
\end{aligned}
$$

The terms in eqns. (3) and (4) with the subscript ' $B$ ' should be interpreted as sum over all the states of the baryonic octets. The meson field equations for the $\sigma, \omega$ and $\rho-$ mesons are then solved self-consistently at a fixed baryon density to obtain the respective field strengths. The EoS for the $\beta$-equilibrated for the hyperon rich matter is obtained with the requirements of conservation of total baryon number and charge neutrality condition given by [23]

$$
\sum_{B} Q_{B} \rho_{B}+\sum_{l} Q_{l} \rho_{l}=0
$$


where $\rho_{B}$ and $\rho_{l}$ are the baryon and the lepton $(\mathrm{e}, \mu)$ number densities with $Q_{B}$ and $Q_{l}$ as their respective electric charges.

Using the computed EoS for the neutron star sequences, we calculate the structural properties of neutron stars with a hyperons core.

\section{STELLAR EQUATIONS}

The equations for the structure of a relativistic spherical and static star composed of a perfect fluid were derived from Einstein's equations by Tolman, Oppenheimer and Volkoff [25], which are

$$
\begin{gathered}
\frac{d P}{d r}=-\frac{G}{r} \frac{[\varepsilon+P]\left[M+4 \pi r^{3} P\right]}{(r-2 G M)}, \\
\frac{d M}{d r}=4 \pi r^{2} \varepsilon
\end{gathered}
$$

with $G$ as the gravitational constant and $M(r)$ as the enclosed gravitational mass. We have used $c=1$. Given an EOS, these equations can be integrated from the origin as an initial value problem for a given choice of central energy density, $\left(\varepsilon_{c}\right)$. The value of $r(=R)$, where the pressure vanishes defines the surface of the star. We solve the above equations to study the structural properties of a static neutron star using the EoS derived for the electrically charge neutral hyperonic dense matter [26, 27.

Although a relativistic compact star has much complicated internal structure but its properties can be reasonably approximated by some simplifying assumptions. The matter inside is assumed to be a perfect fluid on the basis of the observation from pulsar glitches, which shows that the departure from perfect fluid equilibrium due to the solid crust is quite negligible $\left(\sim 10^{-5}\right)[28]$. At birth, a neutron star is differentially rotating, but because of several factors such as the cooling phenomenon, shear viscosity, neutrino diffusion etc., the star assumes a uniform rotation. So the approximation of a zero temperature perfect fluid neutron star matter is a good one.

In order to calculate the models of rotating star basically two approaches are being employed [29], namely the Hartle approach (slow rotation) [30] and the Komatsu, Eriguchi, and Hachisu (KEH) method (fast rotation) [31, 32] approach. Although an improved version of the former approach was employed to calculate the properties of rotating stars [33, 34, 35], large descripancies has been noted compared to models without the assumption of slow rotation, particularly near the mass-shedding limit [36]. In our present investigation, we employ the $\mathrm{KEH}$ approach to calculate the model for rapidly rotating stars near the mass-shedding limit or conversely upto the Kepler frequency, which we briefly describe now.

Let us consider equilibrium stars in uniform rotation with static, axial symmetric space-time. Now the time translational invariant and axial-rotational invariant metric in spherical polar coordinates $(t, r, \theta, \phi)$ can be written as

$d s^{2}=-e^{2 \nu} d t^{2}+e^{2 \alpha}\left(d r^{2}+r^{2} d \theta^{2}\right)+e^{2 \beta} r^{2} \sin ^{2} \theta(d \phi-\omega d t)^{2}$,

where the metric functions $\nu, \alpha, \beta, \omega$ depends only on $r$ and $\theta$. By treating stellar matter as perfect fluid, the energy momentum tensor can be written as

$$
T^{\mu \nu}=P g^{\mu \nu}+(P+\epsilon) u^{\mu} u^{\nu},
$$

with the four-velocity

$$
u^{\mu}=\frac{e^{-\nu}}{\sqrt{1-v^{2}}}(1,0,0, \Omega) .
$$

Here

$$
v=(\Omega-\omega) r \sin \theta e^{\beta-\nu},
$$

is the proper velocity relative to an observer with zero angular velocity and $\Omega$ is the angular velocity of the star measured from infinity. Now we can compute the Einstein field equations given by

$$
R_{\mu \nu}-\frac{1}{2} g_{\mu \nu} R=8 \pi T_{\mu \nu}
$$

(where $R_{\mu \nu}$ is the Ricci tensor and and $R$ is the scalar curvature and with $\mathrm{c}=\mathrm{G}=1$ ). This leads to the equations for the metric functions as

$$
\begin{aligned}
\Delta\left[\rho e^{\frac{\gamma}{2}}\right] & =S_{\rho}(r, \mu), \\
\left(\Delta+\frac{1}{r} \frac{\partial}{\partial r}-\frac{1}{r^{2}} \mu \frac{\partial}{\partial \mu}\right) \gamma e^{\frac{\gamma}{2}} & =S_{\gamma}(r, \mu), \\
\left(\Delta+\frac{2}{r} \frac{\partial}{\partial r}-\frac{2}{r^{2}} \mu \frac{\partial}{\partial \mu}\right) \omega e^{\frac{\gamma-2 \rho}{2}} & =S_{\omega}(r, \mu),
\end{aligned}
$$

where

$$
\Delta=\frac{\partial^{2}}{\partial r^{2}}+\frac{2}{r} \frac{\partial}{\partial r}+\frac{1}{r^{2}} \frac{\partial^{2}}{\partial \theta^{2}}+\frac{1}{r^{2}} \cot \theta \frac{\partial}{\partial \theta}+\frac{1}{r^{2} \sin ^{2} \theta} \frac{\partial^{2}}{\partial \phi^{2}}
$$

$\gamma=\beta+\nu, \rho=\nu-\beta$, and $\mu=\cos \theta$. We refer [31] for the explicit expressions of the source terms $S_{\rho} S_{\gamma}$ and $S_{\omega}$. These basic differential equations can be transformed into an integral representation to handle the boundary conditions in relatively simple manner with the help of multi dimensional Green's functions and solved by KomatsuEriguchi-Hachisu method 31, 32].

A maximum limit for the stable rotation of a star $\Omega_{K}$, is set by the onset of mass shedding from the equator 
of the star. General relativistic expression for this Keplerian frequency ' $\Omega_{K}$ ' can be obtained by using the extremal principle to the circular orbit of a point particle rotating at the equator of the star [37]. This leads to the expression for the Kepler frequency, which is given as,

$$
\Omega_{K}=\omega+\frac{\omega^{\prime}}{2 \psi^{\prime}}+e^{\nu-\beta}\left[\frac{1}{R^{2}} \frac{\nu^{\prime}}{\psi^{\prime}}+\left(\frac{e^{\beta-\nu} \omega^{\prime}}{2 \psi^{\prime}}\right)^{2}\right]^{1 / 2}
$$

where, $\psi=\beta^{\prime}+\frac{1}{R}$, and ' denotes the differentiation with respect to the radial co-ordinate $r$.

Next, the general relativistic expression for the moment of inertia $I$ of a rotating star is given by [38]

$$
I=\frac{1}{\Omega} \int d r d \theta d \phi \sqrt{-g} T_{\phi}^{t}
$$

where $g$ is the determinant of the metric (13). Using eqn. (14-16), the moment of Inertia is then given as,

$$
I=\frac{2 \pi}{\Omega} \int d r \int d \theta r^{3} \sin ^{2} \theta \frac{(P+\epsilon) v}{1-v^{2}} e^{2(\alpha+\beta)},
$$

and hence the angular velocity ' $J$ ' of the star can be obtained from the relation

$$
J=I \Omega .
$$

We use the code written by Stergioulas [39] based on the Komatsu-Eriguchi-Hachisu method to construct uniformly rotating star models.

\section{RESULTS AND DISCUSSIONS}

\section{A. Choice of parameters and compatibility with the Heavy-Ion collision data}

From our earlier work [23] it was conclusive that the effect of varying incompressibility on the underlying nuclear equation of state and the star properties is minimal. There we found that EoS with incompressibilities $K=210,300 \& 380 \mathrm{MeV}$ resulted in stars with nearly same maximum mass at similar central densities. Similar results were obtained in other global properties of the compact star such as gravitational redshift, maximum baryonic mass and radius too. However the difference in nucleon effective mass was found to be prominent both in the dense matter EoS and also on the star properties. We now investigate the effect of varying nucleon effective mass on the rotational attributes of the evolved neutron star using the same model and same set of parameters. It is to be noted that the parameter set is in accordance with recently obtained heavy-ion collision data [40]. Therefore in the present context it shall be interesting to see the implications of the constraints from heavy-ion collision data on neutron star structure.

Here it is imperative to mention that we chose the parameters for the present analysis keeping the incompressibility $(\mathrm{K}=300 \mathrm{MeV})$ constant. The incompressibility is higher in comparison to other successful mean field models like the NL3 $(K=271.5 \mathrm{MeV})$ [4]. However, is well known that the nuclear symmetry energy greatly influences the EoS at high densities. The value for symmetry energy $J=37.4 \mathrm{MeV}$ in case of NL3 is unphysically large in comparison to that we have in the present model $(J=32 \mathrm{MeV})$. On the other hand the DBHF, which is considered to be more realistic EoS in the non-relativistic domain has a symmetry energy of $J=31.5 \mathrm{MeV}$ and is comparable to our prescription and is in good agreement with the empirical models and also with the variational approach [42]. In addition to these aforesaid differences in the nuclear saturation properties, there are variations in the nucleon effective mass in various models. We take $m_{n}^{\star} / m_{n}=(0.80-0.90)$ which is consistent with the values obtained from the analysis of neutron scattering off lead nuclei $[3,43]$.

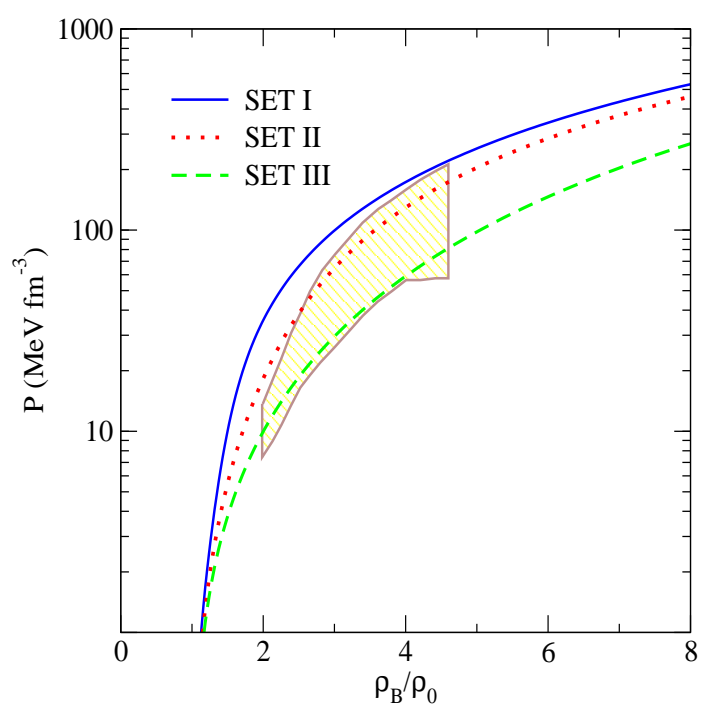

FIG. 1: Pressure $\left(\mathrm{MeV} \mathrm{fm}^{-3}\right)$ as a function of normalized baryon density $\left(\rho_{B} / \rho_{0}\right)$ of symmetric nuclear matter. The shaded region is consistent with the experimental flow data [40].

The parameter set for the present work is listed in Table-1, which vary in nucleon effective masses $\left(m_{N}^{\star} / m_{N}=0.80,0.85 \& 0.90\right)$, but has the same incompressibility value at $K=300 \mathrm{MeV}$. The parameter sets satisfies the nuclear saturation properties, $E_{B}$, energy per nucleon, $-16.3 \mathrm{MeV}$ at saturation density $0.153 \mathrm{fm}^{-3}$, effective nucleon Landau mass $(0.8-0.9) m_{N}$, incompressibility $K=300 \mathrm{MeV}$, and asymmetry energy coefficient value $(\approx 32 \mathrm{MeV})$, so that our extrapolation to 


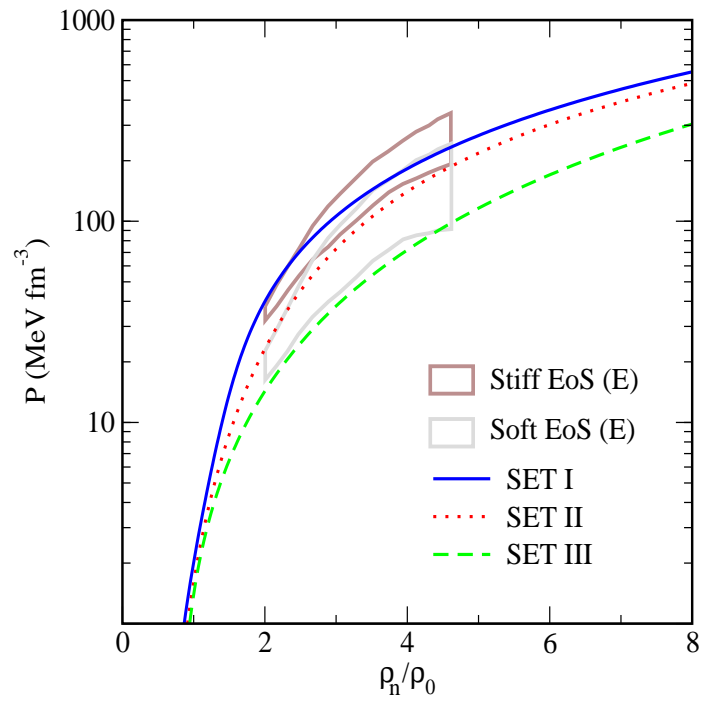

FIG. 2: Pressure $\left(\mathrm{MeV} \mathrm{fm}^{-3}\right)$ as a function of normalized baryon density $\left(\rho_{n} / \rho_{0}\right)$ of neutron matter. The shaded region is consistent with the experimental flow data [40]. The upper polygon represents the stiff EoS, and the lower one corresponds to data for a soft EoS.

higher density remains meaningful. The coupling constant $c_{\rho N}$ is fixed to reproduce $a_{\text {sym }}$ to the empirical value, $32 \pm 6 \mathrm{MeV}$ [4]]. This gives $c_{\rho N}=4.66 \mathrm{fm}^{2}$ for $a_{\mathrm{sym}}=32 \mathrm{MeV}$. The first three columns in table 1 gives the coupling strength of the meson fields and the nucleon, namely the scalar field $c_{\sigma N}$, the vector field $c_{\omega N}$ and the iso-vector coupling strength $c_{\rho N}$, where $c_{i N}=\left(g_{i N} / m_{i}\right)^{2}$, for $i=\sigma, \omega, \rho$. The next two columns are the higher order scalar field constants 'B' and 'C', required to reproduce the bulk nuclear matter saturation properties with reasonable incompressibility which is given in the next column, followed by the nucleon effective mass for the three sets. With these parameterization, $m_{\sigma}=m_{N} \sqrt{\left(c_{\omega N} / c_{\sigma N}\right)}$, turns out to be in the range $(509-627) \mathrm{MeV}$ for the three parameter sets enlisted in Table I. Note that in these calculations, other meson masses or the respective couplings do not enter separately but through the ratio's $c_{\rho N}=\left(g_{\rho N} / m_{\rho}\right)^{2}$ and $c_{\omega N}=\left(g_{\omega N} / m_{\omega}\right)^{2}$.

It is to be noted that the nucleon effective mass taken in the present work lies in the range $m_{N}^{\star} / m_{N}=(0.80-$ 0.90). These values are considerable larger as compared to the Walecka model. A higher effective nucleon mass is known to generate problems in describing finite nuclei properties. However, these values are consistent with the results derived from non-relativistic analysis of scattering of neutrons from lead nuclei [43]. We shall show that the variation in nucleon effective mass shall have considerable impact on the underlying EoS of the dense matter regarding its composition and hence on the gross stellar properties. As stated earlier that the EoS considered for the present study is in accordance with recently obtained
TABLE I: Parameter sets for the model.

\begin{tabular}{cccccccc}
\hline \hline set & $\begin{array}{c}c_{\sigma N} \\
\left(\mathrm{fm}^{2}\right)\end{array}$ & $\begin{array}{c}c_{\omega N} \\
\left(\mathrm{fm}^{2}\right)\end{array}$ & $\begin{array}{c}c_{\rho N} \\
\left(\mathrm{fm}^{2}\right)\end{array}$ & $\begin{array}{c}B \\
\left(\mathrm{fm}^{2}\right)\end{array}$ & $\begin{array}{c}C \\
\left(\mathrm{fm}^{4}\right)\end{array}$ & $\begin{array}{c}K \\
(\mathrm{MeV})\end{array}$ & $m_{N}^{\star} / m_{N}$ \\
\hline I & 8.5 & 2.71 & 4.66 & -9.26 & -40.73 & 300 & 0.80 \\
II & 6.79 & 1.99 & 4.66 & -4.32 & 0.165 & 300 & 0.85 \\
III & 2.33 & 1.04 & 4.66 & 9.59 & 46.99 & 300 & 0.90 \\
\hline
\end{tabular}

heavy-ion collision data. In Fig. 1, we compare our EoS with the experimental flow data from recent heavy-ion collision for symmetric nuclear matter [40]. Here we find that among the three parameter set set II and III are in very good agreement within the entire density domain of $\approx(2-5) \rho_{0}$. Similarly in Fig. 2 , we compare the case for neutron matter with the experimental flow data. The two shaded region correspond to the density dependence of symmetry energy. The upper one corresponds to a strong density dependence (stiff EoS) while the lower one corresponds to a weak density dependence (Soft EoS) of the symmetry energy [40, 45]. Here, all the three EoS seems to be in good agreement with the experimental data. However set I seems represents the stiffest EoS among the three and set III is the softest prescription. Therefore, in the present context, it can be seen that the flow data favors a soft EoS.

\section{B. Composition of Hyperon rich matter}

The composition of charge neutral dense matter is very sensitive to the hyperon-meson coupling parameters which however are very poorly known [15, 46]. There are different phenomenological prescriptions e.g., using the quark counting arguments based on $\mathrm{SU}(6)$ theory to fix the vector couplings, while, fixing the scalar couplings from the potential depths of hyperon in nuclear matter 10, 35, 47]. From various analysis the choice of the ratio of hyperon to nucleon coupling for $\sigma$ meson $x_{\sigma}<0.72$ has been emphasized $[9]$ and also from studies based on hypernuclear levels [48], the choice $\left(x_{\sigma}<0.9\right)$ is bounded from above. Following this convention, in our present work we take $x_{\sigma}=g_{\sigma H} / g_{\sigma N}=0.7, x_{\omega}=g_{\omega H} / g_{\omega N}=0.783$ and $x_{\omega}=x_{\rho}$, to calculate the EOS for the neutron star matter. This leads to the binding of $\Lambda^{0}$ in nuclear matter: $(B / A)_{\Lambda}=x_{\omega} g_{\omega} \omega_{0}+m_{\Lambda}^{*}-m_{\Lambda} \approx-30 \mathrm{MeV}$. We further assume that couplings to the $\Sigma$ and $\Xi$ resonances are equal to those of the $\Lambda$ hyperon [3, 9]. It is worth noticing that taking the $\rho$-meson coupling as $x_{\omega}=x_{\rho}$ or $x_{\sigma}=x_{\rho}$ does not alter the EoS substantially. Further that among all the three coupling schemes, this choice restricts the equation of state of neutron star matter following the constraint of $\Lambda^{0}$ binding in nuclear matter, ably supported by the hypernuclei experiments [16]. We would like to mention here however that the values of these potential are somewhat different [49] from the analysis of $\Sigma^{-}$atomic data and the final state interaction of 
$\Xi$ hyperons in experiments $E 224$ at $K E K[50]$ as well as $E 885$ at $A G S$ [51], which indicates a repulsive potential for $\Sigma^{-}$hyperon in dense matter. However, the exact values of the relativistic potential remain inconclusive. We shall carry out our numerical analysis with the values of the potentials as prescribed above.

As stated earlier, the equation of state of dense matter is very sensitive to the nucleon effective mass. In Fig. 3, we show the respective particle densities of a charge neutral hyperon rich matter for $m^{\star} / m=0.80$ (set I) upto ten times nuclear matter density. Similarly Fig. 4 and Fig. 5 displays the particle population for set II $\left(m^{\star} / m=0.85\right)$ and set III $\left(m^{\star} / m=0.90\right)$ respectively. From the plots, the sensitivity of the nucleon effective mass on the respective particle composition is clearly reflected. It can be seen that with increase in the nucleon effective mass the appearance of various hyperons is pushed further to higher densities. The appearance or concentration of the hyperon species at a particular baryon density is known to regulate the degree of softening effect on the equation of state, which is then manifested in the global properties of compact stars. For example, parameter set I and set II accommodates all the octet of baryons upto ten times normal nuclear matter density although the hyperons starts appearing at higher densities in the later case. However in case of set III, the nucleon chemical potential is not high enough to have $\Sigma^{+}$and $\Xi^{0}$ even upto highest of density region. From the systematics of the nucleon mass in hyperon rich matter, we recall that set II is endowed with repulsive force dominance unlike set III, which is dominated by the scalar forces [23].

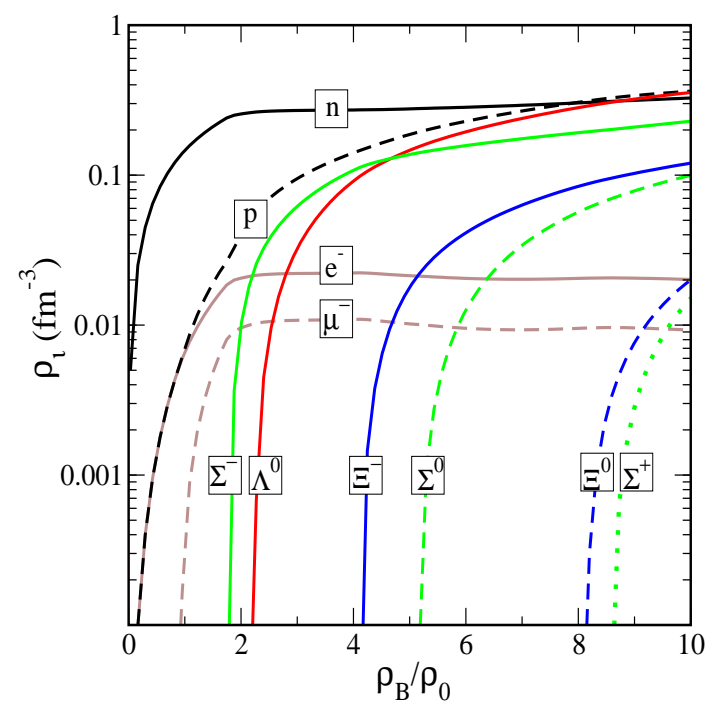

FIG. 3: Particle densities $\left(\mathrm{fm}^{-3}\right)$ in neutron star matter for parameter set I.

In general, for all the parameter sets, the hyperons starts appearing at $\approx 2 \rho_{0}$ and are the dominant species

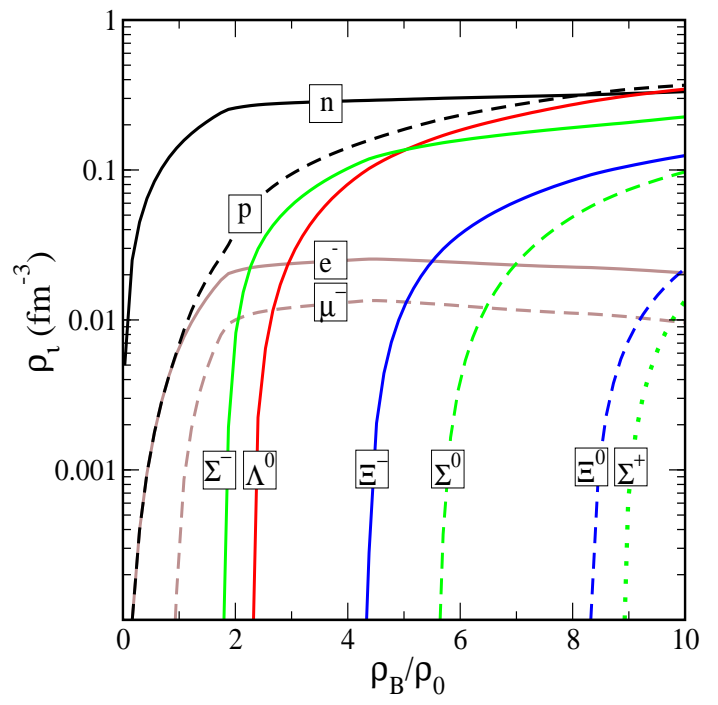

FIG. 4: Same as Fig. 1 but for set II.

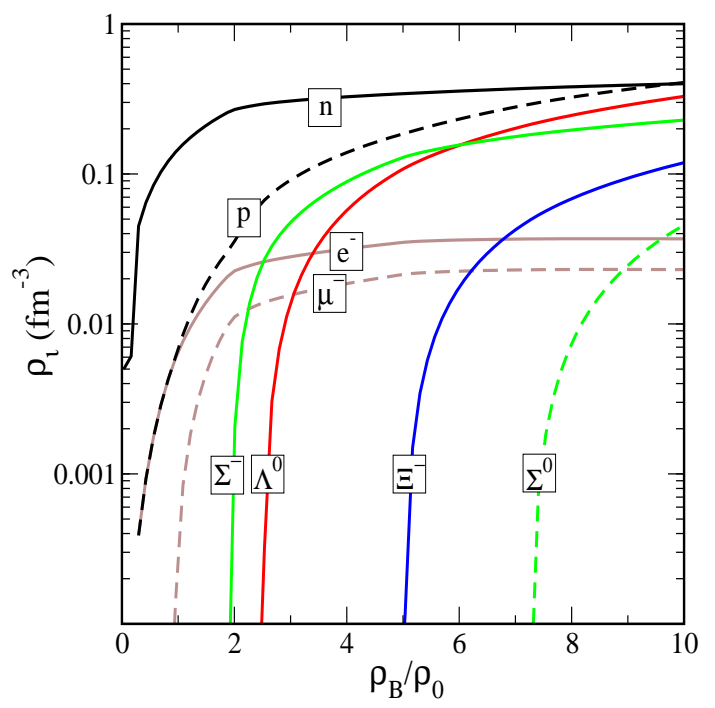

FIG. 5: Same as Fig. 1 but for set III.

in par with nucleons at $\approx 10 \rho_{0}$. It can also be seen that the negatively charged particle species are favored in the dense matter composition followed by the neutral ones and the positively charged counterparts. For example in all the three cases, one finds that $\Sigma^{-}$appears before $\Lambda^{0}$ although the vacuum mass difference between the two is $\approx 77 \mathrm{MeV}$ and the former is massive than the later. This is due to the fact that as the density is increased $\left(\approx 2 \rho_{0}\right)$, the negatively charged $\Sigma^{-}$starts competing with the negatively charged leptons in maintaining charge neutrality of the dense matter. Due to the depletion of the lepton concentration the corresponding chemical potential $\mu_{e}$ decreases, thereby lowering the threshold chemical po- 
tential of $\Sigma^{-}$as compared to $\Lambda^{0}$. Similar arguments exist for the other negatively charged baryons.

\section{Global properties of a rotating Neutron star}

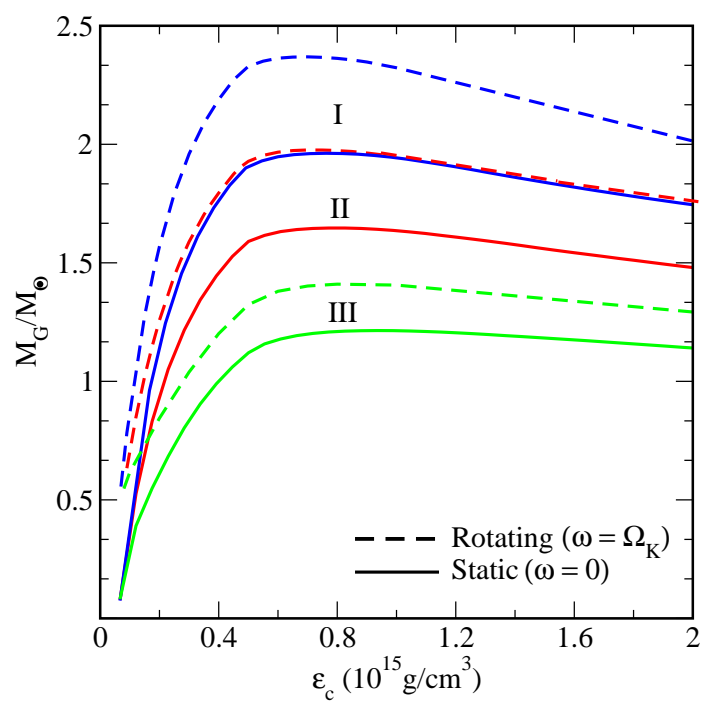

FIG. 6: Dependence of Gravitational mass of the star (in $M_{\odot}$ ) on the central density of the star $\left(10^{15} \mathrm{gcm}^{-3}\right)$ for the three cases in comparison with the static results (solid lines).

The properties of the neutron star is unique to the EoS considered. Using these three EoS we now calculate some of the global properties of the neutron star, which is rotating at its Kepler velocity $\left(\Omega_{K}\right)$ by employing the $R N S$ code [39], as described in section III.

In Fig. 6, we compare the variation of gravitational mass of the star for the three cases as a function of the central density of the star for both the static and the rotating one. In later case, we considered rotation till the onset of mass shedding at the equator or equivalently upto its Kepler frequency. The effect of rotation on the stellar mass is clearly visible from the plot, where it is noticeable that a rotating star acquires more mass than its static counterpart. For all the three case at $\left(\omega=\Omega_{K}\right)$, we notice an increase of nearly $17-20 \%$ in the star mass in comparison to the static ones. However, this increase in mass doesn't alter the central density of the star very much. The maximum mass obtained for set I, II and III, for the rotating case are $2.4 M_{\odot}$, $1.9 M_{\odot}$ and $1.4 M_{\odot}$ respectively and the central densities obtained for both set I and II are $7.0 \times 10^{14} \mathrm{gcm}^{-3}$ and for set III, it is $8.0 \times 10^{14} \mathrm{gcm}^{-3}$. Thus we find that for the three cases the maximum mass is obtained at a central density of $(2.5-3) \rho_{0}$. In case of nonrotating star the central density obtained at maximum mass lies in the range $(3-3.5) \rho_{0}$. The corresponding maximum baryonic masses for set I, II and III for the rotating case are $2.56 M_{\odot}, 2.11 M_{\odot}$ and $1.47 M_{\odot}$ respectively. The difference between the gravitational mass and the baryonic mass gives us the gravitational binding of the star. The fact that at any given density the baryonic mass exceeds the gravitational mass of the star is a typical of compact stars. Consequently, we find that the EoS with lower nucleon effective mass results in more bound compact systems. From the observational point of view, we find that recent observations of neutron star masses like $M_{J 0751 \pm 1807}=2.1 \pm 0.2 M_{\odot}[6]$, $M_{4 U 1636 \pm 536}=2.0 \pm 0.1 \quad M_{\odot}[52], \quad M_{V e l a X-1}=1.86 \pm 0.16$ $M_{\odot}[53]$ and $M_{V e l a X-2}=1.78 \pm 0.23 M_{\odot}[54,55]$ predicts massive stars. Our results are in very good agreement with these observations, except for set III which results in low mass star among the three prescription and has the highest nucleon effective mass value. It is worth to recall that although there are large observational errors in the mass-radius determination, yet in case of VelaX - 1 the lower mass limit $\approx(1.6-1.7) M_{\odot}$ is atleast mildly constrained by geometry [56].

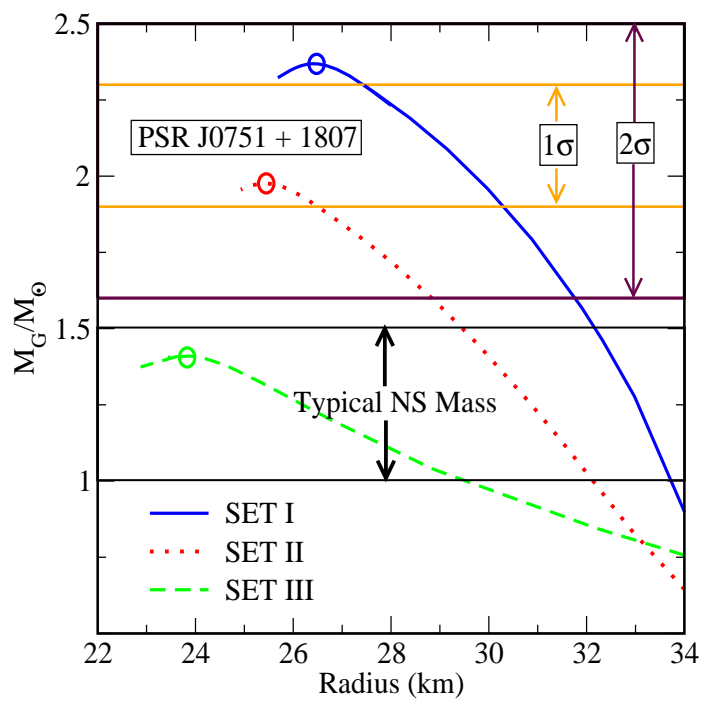

FIG. 7: Gravitational mass of the star $\left(M_{\odot}\right)$ rotating at Kepler frequency as a function of the circumferential radius of the star (in $\mathrm{km}$ ). Also plotted is the typical neutron star mass range $(1.0-1.5) M_{\odot}$ and the mass estimates obtained from PSRJ0751 + 1807 with error estimate of $1 \sigma(2.1 \pm 0.2) M_{\odot}$ and $2 \sigma\left(2.1_{-0.5}^{+0.4}\right) M_{\odot}[\underline{6}]$.

Of the three EoS, in the present analysis, parameter set I is the stiffest prescription and set III is the softest one. This property is well documented in Fig. 7, where we plot the mass of the neutron star as a function of the radius and compare our results with the typical observed mass of neutron stars $(1.0-1.5) M_{\odot}$ and also with more massive ones such as the case of PSR J0751+1807 within the error estimates of $1 \sigma$ and $2 \sigma$. Here we find 


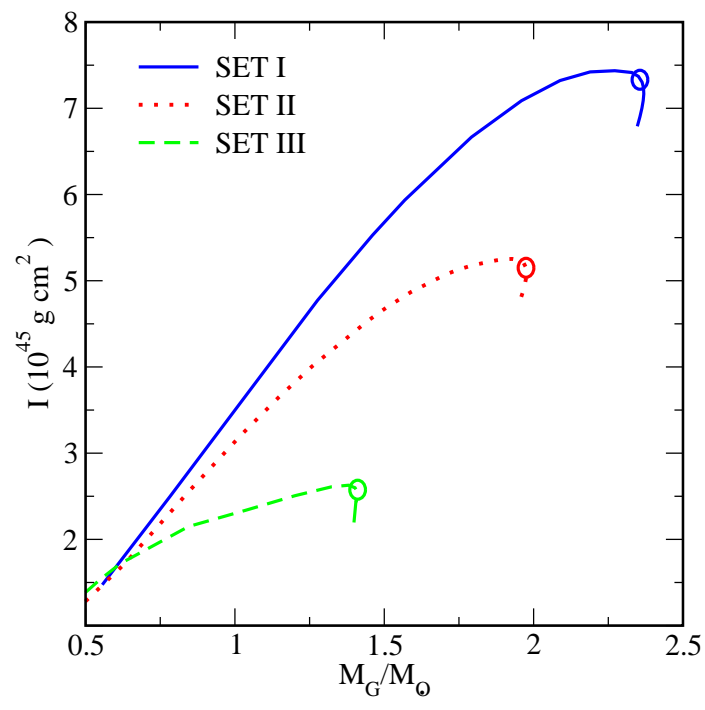

FIG. 8: Moment of Inertia $\left(10^{45} \mathrm{gcm}^{2}\right)$ of the star as a function of the mass of the star. The circled point denotes the value at maximum mass configuration.

that our results are in good agreement with these observations, precisely set II and III agree with the estimates from $P S R J 0751+1807$. In order to evaluate the radius of the star, we have included the BPS EoS [57] at subnuclear densities which contributes to the crustal part of the star structure. The radius obtained for the three EoS lies in the range $(24-26) \mathrm{km}$ and the compactness parameter $(M / R)$ thus lies in the range $(0.05-0.09) M_{\odot} / \mathrm{km}$. From the observational point of view, there are large uncertainties in determination of the radius of the star [58, 59, 60] which is primarily because of our lack of knowledge of the composition of the star atmosphere, large distance and also due to the presence of high magnetic fields. On the other hand, the general relativistic limit for the compactness parameter assuming a uniform density star with the causal equation of state i.e., $P=\varepsilon$ gives $M / R<4 / 9[3]$. In comparison, our prediction lies far below this limit which reflects the softness of our EoS ably supported from the heavy-ion flow data (Fig. 2). Consequently, the flattening parameter, which is defined as the ratio of the polar to the equatorial radius $\left(R_{p} / R_{e}\right)$, for all the three case is $\approx 0.59$, at $\Omega_{K}$. Rotation induces deformation in the shape of the star which leads to a dependence of the star's metric on the polar coordinate $\theta$. In general, rotation stabilizes the star against gravitational collapse and therefore rotating neutron stars are more massive than the static ones. Further, the additional centrifugal forces in a rotating star help to counteract the pull of gravity, resulting in larger radii for a given mass.

Moment of inertia of neutron stars plays crucial role in the models of radio pulsar. Independent of the rotation, i.e., slow or fast, the relation of moment of inertia to the matter distribution within the star is complicated. Among all the global properties of the star, the moment of inertia is the most sensitive one to the dense matter equation of state. In Fig. 8, we show the variation of the moment of inertia of the rotating neutron star as a function of star mass for the three parameter sets. Here it can be seen that set I $\left(m^{\star} / m=0.80\right)$ results in the maximum value of the moment of inertia at $\left(I_{45}=7.22\right.$; $I_{45}=10^{45} \mathrm{gcm}^{2}$ ) and as we move towards the higher effective mass values, this decrease becomes increasingly prominent. But for all the cases we observe that moment of inertia of the compact stars increases rapidly as it approaches the Kepler velocity or till the mass shedding limit. The moment of inertia obtained for set II and III are $I_{45}=5.14$ and $I_{45}=2.54$ respectively. From the observational point of view, recently discovered relativistic pulsar PSRJ0737 - 3039 [61] could be the first one in which the moment of inertia is measured. Also the estimates from crab pulsar [62] puts $I_{45}>1.61$ for $M_{n e b}=2.0 M_{\odot}$, which is the conservative estimate, and $I_{45}>3.04$ for $M_{n e b}=4.6 M_{\odot}$ (latest estimate).

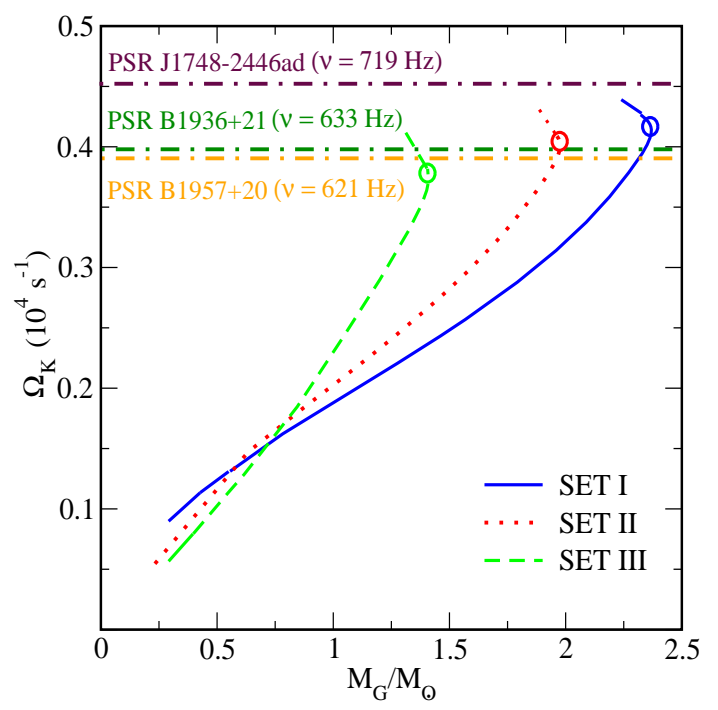

FIG. 9: Keplerian velocity $\Omega_{K}\left(10^{4} S^{-1}\right)$ as a function of the Gravitational mass of the star. The dot-dashed horizontal lines denotes the observed frequency from the pulsars.

In Fig. 9, we plot the Kepler angular velocity $\left(\Omega_{K}\right)$ of the star as a function of the mass of the star. The Kepler angular velocity obtained for the three cases are $4141 s^{-1}, 3999 s^{-1}$ and $3714 s^{-1}$ for set I, II and III respectively, which corresponds to a frequency of $659 \mathrm{~Hz}$, $636 \mathrm{~Hz}$ and $591 \mathrm{~Hz}$. The Kepler period, which is defined as $P_{K}=2 \pi / \Omega_{K}$, obtained are $1.52 \mathrm{~ms}, 1.57 \mathrm{~ms}$ and $1.69 \mathrm{~ms}$ for set I, II and III respectively. It is to be noted that the values that we obtained presently with the three EoS is in agreement with the maximum observed value of the Kepler period for the observed fast rotating pulsars, namely the $P S R B 1937+21(\nu=633 \mathrm{~Hz})$ 63] and PSRB1957+20 $(\nu=621 \mathrm{~Hz})$ [64]. On the other side, 


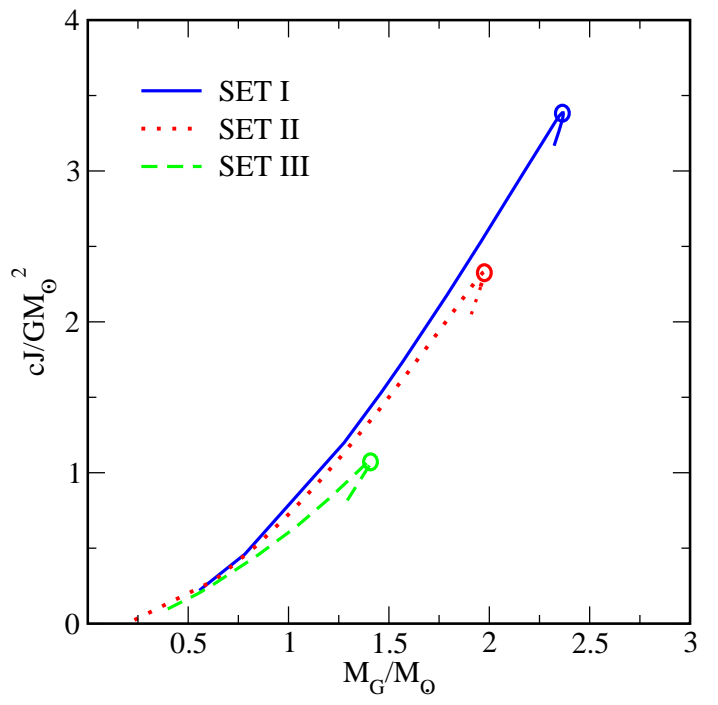

FIG. 10: Angular momentum of the star as a function of the star mass.

the constraint imposed by general relativity on the period of a relativistic compact star emphasize $P>0.24 \mathrm{~ms}[3]$. However there are indications of even faster rotating pulsars from the observational point of view. Recently the observation of the fastest rotating neutron star, with a frequency $\nu=1122 \mathrm{~Hz}$ in an X-ray burst from the X-ray transient, XTE J1739-285 was reported [65] in addition to the observation of $\nu=716 \mathrm{~Hz}$ from $J 1748-2446 \mathrm{ad}$ [66], which may set interesting insight into the structural aspects of neutron stars. It has been mentioned that for the rapid rotation regime, one needs submillisecond pulsars with $\nu>1000 \mathrm{~Hz}$ [67]. However, the smaller $\Omega_{K}$, that we get has to do with the large radii that we obtained in the present EoS. Therefore the present EoS while consistent with the observed PSRB1937+21 and $P S R B 1957+20$ data, could not account for the pulsar rotating with high frequency such as $\nu=716,1122 \mathrm{~Hz}$. This situation seems to be understandable for the fact that the EoS employed presently represents the class of softest EoS in comparison to other theoretical models that can explain the existence of fast rotors. In this context, EoS for skyrmionic models appear to be promising [68]. However, with a phase transition structure possibly having a crystalline color superconducting quark matter may account for the fast rotating pulsars [69], before the mass-shedding limit.

Similar results were obtained for the angular momentum of the star for the three cases, which is shown in Fig. 10. It can be seen that the angular momentum increases rapidly as the star reaches the mass-shed limit and finally attains a maximum value at maximum mass, which is shown with the filled circles for the three cases.

Finally, for a better correlation with the underlying nucleon effective mass, the overall global properties for the static and the rotational case for the three EoS is
TABLE II: Comparison of the static (upper row) and the corresponding rotational (middle row) attributes of the neutron star predicted with the three parameter sets of the present model.

\begin{tabular}{cccccccc}
\hline \hline EoS $m^{\star} / m$ & $M$ & $R_{e q}$ & $\varepsilon_{c} / \varepsilon_{0}$ & $M_{b}$ & $I_{45}$ & $P_{K}$ \\
\hline & & $\left(M_{\odot}\right)$ & $(K m)$ & & $\left(M_{\odot}\right)$ & $\left(10^{45} \mathrm{gcm}^{2}\right)$ & $(m s)$ \\
\hline \hline I & 0.80 & 1.96 & 17.5 & 2.85 & 2.18 & - & - \\
& & 2.36 & 26.4 & 2.60 & 2.56 & 7.22 & 1.52 \\
\hline II & 0.85 & 1.65 & 16.7 & 2.96 & 1.81 & - & - \\
& & 1.98 & 25.5 & 2.60 & 2.11 & 5.14 & 1.57 \\
\hline III & 0.90 & 1.21 & 15.0 & 3.45 & 1.31 & - & - \\
& & 1.41 & 23.7 & 2.96 & 1.47 & 2.54 & 1.69 \\
\hline
\end{tabular}

tabulated in Table 2.

\section{SUMMARY AND OUTLOOK}

In our present work, we intended to present a unified approach to correlate the properties of dense matter EoS with respect to the variation in nucleon effective mass $\left(m^{\star} / m=0.80,0.85,0.90\right)$ defined at saturation density and recently obtained heavy-ion collision data [40]. Subsequently we study the rotational attributes of the sequence of neutron stars in a relativistic framework in the mean-field approach using an effective chiral hadronic model generalized to include all the octet of baryons. The EoS employed in our present investigation satisfies the nuclear matter saturation properties at reasonable incompressibility $(K=300 \mathrm{MeV})$. We then compared the resulting EoS for the three parameter sets with the experimental flow data for both symmetric nuclear matter and neutron matter. We found that all the three parameter sets are in good agreement with the flow data, but on a critical look, parameter set II $\left(m^{\star} / m=0.85\right)$ satisfy these two combined constraint quite well, however all the parameter sets rather lie on the softer domain of neutron matter collision data. The resulting hyperon rich matter was also found to be very sensitive to the difference in the nucleon effective mass. From our analysis, it was conclusive that hyperons form a sizeable population in the dense matter and with higher nucleon effective mass value the threshold for appearance of the respective hyperon species is pushed further to the higher densities. Hyperons are found to have a substantial impact on the density region relevant to neutron star properties and is known to decrease the maximum mass of the star. The ongoing and future experimental hypernuclear programs such as those at Jefferson Lab, KEK, J-PARC and GSI, Darmstadt and FAIR etc. will provide the decisive insights into the role of these exotic forms of matter in dense matter EoS and hence neutron stars, so as to efficiently constrain the dense matter EoS.

We calculated the global properties of the rotating neutron star sequences such as the mass, radius, central den- 
sity, moment of inertia and Kepler angular velocity in the KEH method or the fast rotation approach. The maximum mass obtained at Keplerian velocity for the three sets lies in the range $(1.4-2.4) M_{\odot}$ which is in very good agreement with recent observations of massive neutron stars such as $P S R J 0751+1807$, although we do find substantial increase in the star radius. On overall analysis, parameter set II seems to fit the observed mass of PSR J0751 + $1807(2.1 \pm 0.2)$ within $1 \sigma$ error bar, which can be considered as ideal parameterization in the present context. The compactness parameter $(M / R)$ that we obtained for the three EoS represents the lower limit from the observational point of view. The corresponding central density at maximum mass lies in the range $(2.6-3.0) \rho_{0}$. The flattening parameter i.e., the ratio of the polar radius to the equatorial radius obtained for the three sets is $\approx 0.59$ which reflects the degree of deformation that can result in case of rapidly rotating stars. Subsequently the circumferential radius obtained for the three cases lies in the range $(23.7-26.4) K m$. Further we obtained the Kepler period in the range $(1.52-1.69) \mathrm{ms}$, which can account for the fast pulsars observed such as the case of $P S R B 1937+21$ and $P S R B 1957+20$, rotating with frequency of $\nu=633 \mathrm{~Hz}$ and $\nu=621 \mathrm{~Hz}$ respectively. However our EoS could not explain the pulsars rotating beyond $\nu=659 \mathrm{~Hz}$, keeping in mind that we investigated the neutron star sequences with a hyperon core. Possibly in order to account for pulsars rotating at still higher frequencies, we require a stiff EoS with a phase transition structure in the neutron star core as emphasized in Ref [69].

The observation of high mass stars has raised considerable amount of debate over the nature of EoS i.e., the stiffness or softness of the EoS at high densities. In the present context we would like to stress that the EoS obtained in the present model belongs to the softest prescription in comparison to other theoretical models, as also evident from the analysis with respect to the experimental flow constraint. We also want to emphasize that the present EoS is compatible with the DBHF at low density and the phenomenological RMF-NL3 parameterization at high densities [22]. Besides, the analysis in the present work throws interesting insights with regard to the implications of heavy-ion collision data on the neutron star structure. Here we see that parameter set I and III represents the upper and the lower bound of the flow data in the symmetric nuclear matter case, although all the three sets satisfies the soft prescription of the neutron matter domain. Apparently this was also evident from the global properties of the star that we obtained in our present analysis. The present EoS results in small Kepler frequency before the mass-shedding limit which is for the fact that we obtain a large circumferential radii for the neutron stars in the present model, which greatly influences the rotational attributes of the star. However we would like to mention here that more systematic calculations are required before we arrive at some precise conclusions on the constraints of dense matter EoS and neutron star properties provided by the present model. We explored here the model of neutron star with a hyperon core, it shall be interesting to study and analyze these results including the phase transition aspects such as the transition to superconducting quark matter in the neutron star core, which we shall take up in our next endevour [70].
[1] T. Klähn et. al., Phys. Rev. C 74, 035802 (2006).

[2] S. L. Shapiro and S. A. Teukolski, Black holes, white dwarfs, and Neutron stars (Wiley, New York, 1983);

[3] N. K. Glendenning, Compact Stars: Nuclear Physics, Particle Physics, and General Relativity, Springer Verlag, 2000.

[4] G. Baym, nucl-th/0612021 in AIP Conf. Proc., 892 8, (2007).

[5] S. M. Ransom et al., Science 307, 892 (2005).

[6] D. J. Nice, et al. Astrophys. J. 634, 1242 (2005).

[7] A. J. Faulkner et al., Astrophys. J. L 618, L119 (2004).

[8] J. M. Weisberg, and J. H. Taylor, ASP Conf. Ser. 328, Binary Radio Pulsars, eds. F. A. Rasio and I. H. Stairs, Ast. Soc. Pac., San Francisco, 25 (2005); astro-ph/0407149.

[9] N.K. Glendening and S.A. Moszkowski, Phys. Rev. Lett. 67, 2414 (1991).

[10] J. Schaner-Beilich and I.N. Mishustin, Phys. Rev. C 53, 1416 (1996).

[11] M. G. Alford, K. Rajagopal and F. Wilczek, Nucl. Phys. B 537, 443 (1999).

[12] F. Weber, Prog. Part. Nucl. Phys. 54, 193 (2005).

[13] J. M. Lattimer and M. Prakash, Astrophys. J. 550, 426
(2001).

[14] N. K. Glendening, Phys. Lett. B114, 392 (1982); N. K. Glendening, Astrophys. J. 293, 470 (1985); N. K. Glendening, Z. Phys. A 326, 57 (1987).

[15] M. Prakash, I. Bombaci, M. Prakash, P.J. Ellis, J.M. Lattimer and R. Knorren, Phys. Rep. 280, 1 (1997).

[16] D. J. Millener, C. B. Dover and A. Gal, Phys. Rev. C 38, 2700 (1988).

[17] H. Müller and B. D. Serot, Nucl. Phys. A 606, 508 (1996).

[18] M. Prakash and T. L. Ainsworth, Phys. Rev. C 36, 346 (1987).

[19] N. K. Glendening, Nucl. Phys. A 480, 597 (1988).

[20] P. K. Sahu, R. Basu, and B. Datta, Astrophys. J. 416, 267 (1993).

[21] P. K. Sahu and A. Ohnishi, Prog. Theor. Phys. 104, 1163 (2000).

[22] P. K. Sahu, T. K. Jha, K. C. Panda, and S. K. Patra, Nucl. Phys. A 733, 169 (2004).

[23] T. K. Jha, P. K. Raina, P. K. Panda and S. K. Patra, Phys. Rev. C 74, 055803 (2006); Erratum- Phys. Rev. C 75029903 (2007).

[24] B. D. Serot and J. D. Walecka, Adv. Nucl. Phys. 16, 1 (1986). 
[25] J. R. Oppenheimer and G. M. Volkoff, Phys. Rev 55, 374 (1939); R. C. Tolman, Phys. Rev 55, 364 (1939).

[26] J. M. Lattimer, and M. Prakash, Phys. Rept., 333, 121 (2000); J. M. Lattimer, and M. Prakash, Science, 304, 536 (2004).

[27] P. G. Krastev, and F. Sammarruca, Phys. Rev., C 74 025808 (2006); H. Heiselberg, and V. Pandharipande, Ann. Rev. Nucl. Part. Sci., 50, 481 (2000).

[28] J. L. Friedman and J. R. Ipser, Philos. Trans. R. Soc. London, A 340, 391 (1992).

[29] J. L. Friedman, Proceedings of the 1994 Aspen Conference om Millisecond Pulsars, edited by A. S. Fruchter et. al. (Astronomical Society of the Pacific Conference Series), vol 72, 177 (1995).

[30] J. B. Hartle, Astrophys. J., 150, 1005 (1967).

[31] H. Komatsu, Y. Eriguchi and I. Hachisu, Mon. Not. R. Astron. Soc. 237, 355 (1989).

[32] H. Komatsu, Y. Eriguchi and I. Hachisu, Mon. Not. R. Astron. Soc. 239, 153 (1989).

[33] B. Datta, Fundam. Cosmic Phys., 12, 151 (1988); F. Weber, and N. K. Glendenning, Phys. Lett. B, 265, 1 (1991); F. Weber, and N. K. Glendenning, Astrophys. J., 390, 541 (1992).

[34] A. Gupta, A. Mishra, H. Mishra, and A. R. Prasanna, Class. Quant. Grav. 15, 3131 (1998); S. Schramm, and D. Zschiesche, J. Phys. G 29, 531 (2003).

[35] A. Mishra, P. K. Panda, and W. Greiner, J. Phys. G 28, 67 (2002).

[36] M. Salgado, S. Bonazzola, E. Gourgoulhon, and P. Haensel, Astron. Astrophys., 291, 155 (1994).

[37] N.K. Glendening and F. Weber, Phys. Rev. D 50 (1994) 3836 .

[38] J.B. Hartle, Astrophys. Space Sci. 24, 385 (1973).

[39] N. Stergioulas and J. L. Friedman, Astrophys. J. 444, 306 (1995).

[40] P. Danielewicz, R. Lacey, W.G. Lynch, Science 298, 1592 (2002).

[41] G. A. Lalazissis, J. König, and P. Ring, Phys. Rev. C 55, 540 (1997); S. Typel, Phys. Rev. C 71064301 (2005).

[42] A. Akmal, V. R. Pandharipande, and D. G. Ravenhall, Phys. Rev. C 58, 1804 (1998).

[43] C. H. Johnson, D. J. Horen and C. Mahaux, Phys. Rev. C 36, 2252 (1987).

[44] P. Möller, W.D. Myers, W.J. Swiatecki and J. Treiner, At. Data Nucl. Data Tables 39, 225 (1988).

[45] M. Prakash and T. L. Ainsworth, Phys. Rev. Lett. 61, 2518 (1988).

[46] N. K. Glendening Phys. Rev. C 64, 025801 (2001).

[47] J. Schaffner, C. B. Dover, A. Gal, D. J. Millener, C. Greiner and H. Stöcker, Ann. Phys., NY 23535 (1994);
S. Banik and D. Bandyopadhyay, Phys. Rev. C 63035802 (2001); C. B. Dover, D. J. Miller, and A. Gal, Phys. Rep. 1841 (1989).

[48] M. Rufa, J. Schaffner, J. Maruhn, H. Stocker, W. Greiner and P. G. Reinhard, Phys. Rev. C 42, 2469 (1990).

[49] J. Schaffner-Beilich and A. Gal, Phys. Rev. C 62034311 (2000).

[50] T. Fukuda et. al., Phys. Rev. C 581306 (1998).

[51] P. Khaustov et. al., Phys. Rev. C 61054603 (2000).

[52] D. Barret, J.F. Olive and M.C. Miller, astro-ph/0605486

[53] O. Barziv, et. al., Astron. Astrophys. 377, 925 (2001).

[54] J. Casares, P.A. Charles and E. Kuulkers, Astro. J. 493 L39 (1998).

[55] J. A. Orosz, E. Kuulkers, Mon. Not. R. Astron. Soc. 305, 132 (1999).

[56] H. Quaintrell et al., Astron. and Astrophys. 401, 303 (2003).

[57] G. Baym, C. Pethick and P. Sutherland, Astrophys. J. 170, 299 (1971).

[58] R. Rutledge, L. Bildsten, E. Brown, G. Paplov and V. Zavlin, Astrophys. J. 577, 346 (2002); Astrophys. J. 578, 405 (2002).

[59] B. Gendre, D. Barret and N. A. Webb, Astron. and Astrophys. 400, 521 (2003); W. Becker et. al., Astrophys. J. 594, 364 (2003).

[60] J. Cottam, F. Paerels and M. mendez, Nature 420, 51 (2002).

[61] A. G. Lyne et. al., Science 303, 1153 (2004).

[62] M. Bejger and P. Haensel, Astron. Astrophy. 396, 917 (2002).

[63] D. C. Backer, S. R. Kulkarni, C. Heiles, M. M. Davis, and W. M. Goss, Nature 300615 (1982).

[64] A. S. Fruchter, D. R. Stinebring, and J. H. Taylor, Nature 334237 (1988).

[65] P. Kaaret et. al; astro-ph/0611716.

[66] J. W. T. Hessels, S. M. Ransom, I. H. Stairs et, al., Science, 311, 1901 (2006).

[67] G. B. Cook, S. L. Shapiro, S. A. Teukolsky, Astrophys. J., 424, 823 (1994); M. Salgado, S. Bonazzola, E. Gourgoulhon and P. Haensel, Astron. \& Astrophys. 108, 455 (1994).

[68] Prashanth Jaikumar, Manjari Bagchi, and Rachid Ouyed, astro-ph/0708.3436

[69] Nicola D. Ippolito1, Marco Ruggieri1, Dirk H. Rischke, Armen Sedrakian, and Fridolin Weber, astroph/0710.3874.

[70] T. K. Jha, H. Mishra and V. Sreekanth, (under preparation). 